\title{
Philippe Poirrier (ed.), La Historia Cultural ¿un giro historiográfico mundial? Valencia: Universidad de Valencia, 2012, 252 págs.
}

El presente volumen se presenta ante el lector avezado como un manual académico de historiografía abocado a la reflexión de los estudios culturales a escala mundial. Bajo la edición de Philippe Poirrier (Université de Bourgogne) un grupo de catorce colaboradores parten de su situación historiográfica nacional y analizan las modalidades de surgimiento y de estructuración de la historia cultural. En términos generales, en los diferentes capítulos se combina el análisis de los textos clásicos en la materia, el estudio de las coyunturas historiográficas específicas a cada contexto nacional, la organización de los mercados universitarios, el impacto de los estudios culturales y la importancia de las transferencias exteriores en la asimilación de los modelos historiográficos.

En el 2008, Poirrier publicó una primera edición de este texto en lengua francesa. ${ }^{1}$ Dos años después, Alessandro Arcagelli tuvo a su cargo la edición italiana del texto. ${ }^{2} \mathrm{Al}$ presente, el texto que reseñamos - publicado en 2012- ofrece la ventaja de combinar ambas obras y presentarlas traducidas al español a la vez que dedica dos capítulos nuevos dedicados a Alemania y los Países Bajos. Ambos capítulos - a cargo de Xenia von Tippelskirch y Herman Roodenburg, respectivamente- no se incluyeron en la edición original francesa dado que los autores no cumplieron con los plazos de tiempo establecidos en aquel momento. En consecuencia, la edición que nos ocupa tiene la particular virtud de ofrecer una perspectiva mundial más completa que las anteriores.

Tanto en la presentación del libro - a cargo de los españoles Justo Serna y Anaclet Pons- como en la introducción del propio editor de la obra y en epílogo de Roger Chartier, se puede leer una vehemente defensa de la historia cultural puesto que, aun cuando hay fuertes indicios de su éxito en los últimos años, todavía es objeto de diversas críticas en ciertos cenáculos académicos. Algunos le recriminan la indefinición de sus objetos y su transformación en un inventario interminable que enumera prácticas cotidianas; otros sostienen que desatiende las determinaciones más fundamentales que gobiernan las relaciones y las desigualdades sociales. A la primera imputación, Chartier responde que "más allá de la diversidad de sus temas y enfoques, la historia cultural se ha organizado a partir de algunos interrogantes fundamentales: las relaciones entre cultura popular y cultura de élite, la articulación entre las representaciones comunes y las obras singulares y las relaciones entre discursos y prácticas, entre lenguaje y experiencia” (p. 252). Es decir, sin necesidad de imponer un único paradigma, estos elementos compartidos le dan a la historia cultural una consistencia teórica y metodológica innegable. Por otra parte, ante la segunda acusación los autores advierten que justamente han sido los historiadores y los sociólogos quienes más importancia le otorgaron para detectar los mecanismos que instituyen y reproducen las dominaciones, y quienes han determinado el alcance de las representaciones mentales en la construcción del mundo social. La historia cultural se ocupa de las prácticas

\footnotetext{
${ }^{1}$ Philippe Poirrier, L'histoire culturelle: un tournant mondial de l'historiographie? (Dijon: EUD, 2008).

2 Alessandro Arcangelli, La storia culturale: una svolta nella storiografia mondiale? (Verona: QuiEdit, 2010).
}

ISSN 2174-4289 
y las representaciones pero no surge para sustituir la investigación académica ni es contraria a ella. En términos de Peter Burke, surge para complementarla con tres objetos: la historia de las representaciones, la historia del cuerpo y la historia cultural de la ciencia. ${ }^{3}$ ¿Temas sin importancia? Los historiadores de la cultura han respondido que allí no reside el problema, puesto que el objeto de estudio no determina ni el rango ni la calidad del estudio dado que todo depende de la manera en que se trata el fenómeno (p. 185).

En el capítulo dedicado a la historia cultural en Gran Bretaña, Peter Burke encuentra que, a pesar de la importancia de la obra de Raymond Williams, ${ }^{4}$ existe una tradición de individualismo metodológico y un recelo respecto de los conceptos imprecisos como el propio de "cultura". El autor analiza la historia de la historia cultural de la primera mitad del siglo XX y encuentra que las aportaciones británicas en historia cultural fueron poco significativas. ${ }^{5}$ La segunda parte del ensayo se refiere al periodo 1950-1980, dominado por los historiadores sociales marxistas. En tiempos de la Guerra Fría, se destacan las contribuciones al dominio de la historia cultural de los marxistas Joseph Needham, Edward Thompson y el ya citado Raymond Williams. En el ámbito de los estudios medievales, la obra más destacable de historia cultural en este periodo fue The Making of the Middle Ages (1953) de Richard Southern. Al mismo tiempo, se destaca el trabajo de los antropólogos y se reconocen los aportes de Edward Evans-Pritchard, inspirador de Keith Thomas en Religion and the Decline of Magic (1971). La última parte se ocupa de la época del giro cultural iniciado en la década de 1980. Nuevamente, se hace presente el nombre de K. Thomas pero unido a los de Gareth Stedman Jones, Benedict Anderson, Eric Hobsbawm, Terence Ranger, entre otros. En los últimos 30 años, los ingleses han entrado de lleno en la globalización cultural evidenciándose un declive de la "cultura del empirismo".

En Francia, la historia cultural se estructuró también en los años ochenta y noventa pero con la particularidad de que aparece como una forma de historia social que la diferencia de la Cultural History norteamericana y de los trabajos que remiten al Lingüistic Turn y a los Cultural Studies. El nombre de Roger Chartier se destaca en el panorama historiográfico francés a partir de su trabajo más memorable. ${ }^{6}$ En los debates que se dieron en Francia alimentados por la aparición del "giro lingüístico" Chartier ocuparía un rol esencial en tanto defensor de la historia cultural. Ante la imputación de que la historia no era más que un género literario como cualquier otro, el historiador de la cultura subrayó que la fundamental diferencia entre literatura e historia estaba mediada por la exigencia de verdad demostrada que se basaba en un método científico.

El análisis del panorama de los estudios italianos en materia de historia cultural se encargó a Alessandro Arcangeli, historiador modernista de la Università de Verona y

\footnotetext{
${ }^{3}$ Peter Burke, “Cultural History as Polyphonic History”, Arbor, 743 (2010): 479-86.

${ }^{4}$ Raymond Williams, Culture and Society 1780-1950 (London: Chatto and Windus, 1958).

${ }^{5}$ Las excepciones se hacen presents en los nombres de F. R. Leavis (Mass Civilization and Minority Culture); B. Willey (The Seventeenth Century Background); E.M.W. Tillyard (Elizabeth World Picture); T.S. Eliot (Notes Towards the Definition of Culture); N. Elías (The Civilizing Process); C. Dawson (Enquiries into Religión and Culture); y A. Toynbee (Study of History).

${ }^{6}$ Roger Chartier, El mundo como representación. Historia cultural: entre práctica y representación (Barcelona: Gedisa, 1992). Si bien citamos la edición en español por motivos prácticos, recordamos al lector que los trabajos de Chartier sobre las singularidades de la historia cultural se remontan a la década de 1980.
}

ISSN 2174-4289 
encargado de la compilación italiana de 2010 a la que ya hemos hecho referencia. El autor presenta un texto más descriptivo que analítico para poner de manifiesto que la singularidad italiana reside en el hecho de que, si dejamos de lado la problemática noción de historia cultural, podremos apreciar que el estudio de los aspectos culturales en la historia es un aspecto muy arraigado en la historiografía nacional. Este ensayo, al igual que el consagrado a la historia cultural en Brasil a cargo de Andréa Daher, adolece de la ausencia de un cierre conclusivo que permita hacer un balance de las perspectivas historiográficas nacionales en su evolución histórica hasta la actualidad.

El caso norteamericano es trabajado por Edward Berenson que, ante la inmensidad del tema propuesto -historia cultural en los Estados Unidos-, decide inteligentemente tomar como estudio de caso la historia cultural de Francia realizada desde los Estados Unidos. Este es un estudio de historia cultural de Francia à la américaine. Durante los años sesenta, la dimensión marxista de la historiografía francesa conquistó a un gran número de jóvenes historiadores norteamericanos que dominaban el idioma francés y decidieron explorar los dominios de la historia social. Las figuras de Thompson, Agulhon, Geertz y Darnton ejercieron un magisterio determinante en la historiografía norteamericana. Paulatinamente, la cuestión del "género" y el estatus del discurso se instalaron en el núcleo de la historia cultural americana. En los últimos años, los especialistas se han inclinado por el problema de las relaciones entre historia y memoria a la vez que se interesan por el Imperio, la colonización y por las cuestiones de identidad racial y étnica (p.75). En relación al tema de la identidad, Carl Bouchard sostiene que en Canadá los trabajos históricos tratan fundamentalmente sobre la formación de la identidad colectiva (p.143). A esta temática tan frecuentada en los estudios culturales canadienses hay que sumar las siguientes líneas de investigación: la historia del libro y de la imprenta, la vida literaria y los medios de comunicación, la historia cultural de lo religioso, la conmemoración y la construcción del pasado (pp. 151-163).

El ensayo de Palle Ove Christiansen consagrado a la historia cultural en Escandinavia presenta un rasgo de originalidad que lo distingue. El autor no solamente pone el acento en una lista de autores y títulos - como han hecho el resto de los colaboradores del volumensino que también propone perspectivas de corte más netamente historiográfico. En Escandinavia, la historia cultural representó "una reacción con respecto al modernismo empirista de la historia oficial" (p.79). Surge en rebeldía a una historia política decimonónica creadora de conciencia nacional pan-nórdica respecto a la Europa de Bismarck y a una historia social cuantitativa. Desde este nuevo enfoque, muchos historiadores han incursionado en nuevos temas mientras que otros siguen abordando problemáticas tradicionales pero desde un nuevo ángulo.

El capítulo referido a la historia cultural y los historiadores culturalistas en Australia demuestra los avances que tuvieron lugar en este terreno en los últimos cuarenta años en lo que se refiere a su integración en la comunidad científica internacional. Martyn Lyons presenta el debate en el contexto de la profesión de historiador en su conjunto en Australia para abordar a continuación la historia cultural a través de tres áreas principales: las 
supuestas querellas de los historiadores, ${ }^{7}$ la nueva historia del Imperio británico, ${ }^{8}$ y el trabajo de la Escuela de Melburne. ${ }^{9}$ El análisis estadístico de los artículos publicados en Australian Historical Studies en el período 1996-2005 permite apreciar el estatus de la historia cultural, destacándose el área de estudios de historia de los géneros.

La reflexión del espacio historiográfico español fue encargado a Pons y Serna, especialistas en esta área que cuentan con una valiosa trayectoria en la materia que resumen para este volumen. ${ }^{10}$ Los autores destacan que en España hay muchas obras que se presentan a sí mismas como historia cultural pero que es necesario establecer un rango y una categoría entre dichos textos fijando una jerarquía de los objetos a partir de la calidad de los autores, la frecuencia con que se repiten y su influencia en los otros investigadores. En los últimos años, las editoriales españolas han publicado varios ensayos historiográficos que recuperan a los clásicos de la historia cultural (Burckhardt, Huizinga, Warburg, Elías, Gombrich, Panofsky) que más han orientado a los representantes actuales más significativos. Entre los historiadores culturales contemporáneos el nombre de Chartier constituye una vez más una cita obligada en España. Pons y Serna afirman que "la historiografía francesa ha tenido siempre la máxima influencia en España” (p.190). Este hecho, sumado a la personalidad y el excelente dominio del español y la cultura hispanoamericana de Chartier, hace que el impacto de su obra en la Península Ibérica no nos sorprenda. Como ha señalado Ricardo García Cárcel, "el presente de la nueva historia de la cultura tiene un nombre propio, Roger Chartier". "Asimismo, la obra de Burke ha influido -aunque en menor medida que Chartier- en los trabajos de algunos modernistas como James Amelang y sus discípulos. A diferencia de España donde los historiadores de la cultura se han ocupado mucho de la teoría, la historiografía holandesa carece de "grandes y amplias perspectivas, que a menudo se dejan a los estudios extranjeros que se ocupan de los Países Bajos" durante la Edad Moderna (p. 244).

Las nuevas perspectivas de historia cultura en Suiza se hallan presentes en infinidad de trabajos de investigación pero su institucionalización -definida por la existencia de autores, obras, cátedras, programas de doctorado, revistas específicas- resulta incompleta y de escasa consistencia. Esta situación no es privativa del caso suizo puesto que en Bélgica se observa una situación similar. Esto no significa que la disciplina sea ajena a las preocupaciones de los investigadores puesto, que se han publicado trabajos de enormes valor. Incluso en España, Pons y Serna sostienen que la institucionalización de la historia

\footnotetext{
${ }^{7}$ Polémica politizada sobre las versiones de la cultura de la identidad nacional. Por un lado, los conservadores sostienen que la historia australiana es positiva, optimista, justa, igualitaria, progresiva y marcada por la tolerancia. Por otra parte, son numerosos los historiadores que subrayan que la cara oscura del éxito viene dada por la violencia en la "frontera" que implicó la confiscación a los indígenas en el contexto de un racismo institucionalizado cargado de misoginia.

${ }^{8}$ Se refiere a la escritura de una historia transnacional que solucione el problema del australocentrismo en la historiografía nacional. Los historiadores de la cultura han realizado importantes aportes al respecto estudiando por ejemplo las redes de conocimientos, las influencias culturales recíprocas, etc.

${ }^{9}$ Esta Escuela está constituida por un grupo de historiadores australianos que estudian la transmisión cultural y los encuentros entre los sistemas culturales de las Américas y del Pacífico.

${ }^{10}$ Justo Serna y Anaclet Pons, La historia cultural. Autores, obras, lugares (Madrid: Akal, 2005).

${ }^{11}$ Ricardo García Cárcel, “Aproximación a la historia de la cultura en España a lo largo del siglo XX”, Revista de Historia Jerónimo Zurita, 71(1995): 36.
}

ISSN 2174-4289 
cultural todavía se presenta incompleta fundamentalmente entre los especialistas de historia contemporánea. $^{12}$

En el otro extremo, la historia cultural alemana "no solo goza hoy en día de un auge considerable en el mundo editorial, sino que ha sido institucionalizada con la creación de algunas cátedras universitarias" (p.217). El capítulo sobre Alemania aborda el proceso de institucionalización y una síntesis de los debates que han nacido en torno a la Kulturgeschichte. Xenia von Tippelskirch destaca que la nueva historia cultural en la década de 1980 nació a partir del enfrentamiento la nueva historia social, esta última fuertemente influida tanto por Annales como por las tendencias anglosajonas. Se trataba de una historia social que desafiaba a la narración de tipo historicista aspirando a la comprensión de la sociedad en su conjunto. Los nuevos historiadores de la cultura criticaron aquella pretensión de exhaustividad. Los partidarios de la historia de lo cotidiano, promovida por el Max-Planck-Institut für Geschichte de Gotinga, criticaron a los historiadores sociales sus presupuestos más esenciales. La historia, para ellos, no era el resultado de circunstancias estructurales, sino el efecto de prácticas culturales de individuos y grupos. Asimismo, llamaban la atención sobre el estudio de la gente común y tomaron las propuestas de la microhistoria para centrarse en realidades locales y regionales.

En Rumania, la historia cultural atravesó diversas etapas: época clásica con Burckhardt y Huizinga, época de Annales con Bloch y Febvre y época de la tercera generación de Annales tras la segunda postguerra. Durante el periodo comunista, aun cuando se imponía a los historiadores una perspectiva marxista y sobre todo estalinista, hubo autores que decidieron escribir sobre la cultura. Ecaterina Lung, medievalista rumana, sostiene que aun cuando la historia cultural no era un tema preferente, después de 1965 volvió a ser un tema legítimo. ${ }^{13}$ Generalmente se estudiaba más la Antigüedad, la Edad Media y los comienzos de la modernidad. Con la caída del régimen comunista en 1989, la historiografía rumana recibiría - no sin recelos y con un proceso paulatino que todavía daba preferencia a la historia política- las nuevas teorías y metodologías de Occidente.

Una lectura transversal de los distintos ensayos demuestra que cada una de las tradiciones nacionales ha asimilado, en distintos momentos y contemplando diversas formas, las proposiciones procedentes de otras historiografías. En la mayoría de los casos estudiados, la historia cultural estuvo marginada durante el siglo XIX por presentarse incompatible con los criterios historicistas vigentes que se centraban en cuestiones políticas y en la apertura a los intereses a las naciones emergentes. Descontando algunas excepciones puntuales, quizá el más famoso de los historiadores culturales del siglo XIX fue Burckhardt. Estos investigadores fueron acusados a menudo de diletantismo. Habría que esperar hasta los años ochenta del siglo XX para que la historia cultural se estructurara; $\mathrm{y}$

\footnotetext{
${ }^{12}$ Véase Manuel Peña, "La historiografía francesa en la historia cultural de la Edad moderna española. Breve balance de su influencia”, en Benoît Pellistrandi (ed.), La historiografía francesa del siglo XX y su acogida en España (Madrid: Casa de Velázquez, 2002) 177-88.

${ }^{13}$ Se destaca la obra del historiador rumano Alexandru Dutu, quien se adhirió a la Escuela de Annales en diferentes artículos: "Mentalité, durée et le paradigma des Annales", Revue Roumaine d'Histoire XVIII, 1 (1979); y Dimensiunea umană a istoriei. Direcții în istoria mentalităților (Bucarest: Editura Meridiane, 1986).
}

ISSN 2174-4289 
hasta los noventa para que tomase forma y comenzase el auge al que nos hemos referido que contempla variaciones regionales- en distintos países. Los dos grandes modelos historiográficos han sido los Annales (historia de las mentalidades, antropología histórica, historia sociocultural) y una historiografía anglosajona procedente de un marxismo heterodoxo presente en los Cultural Studies, el Lingüistic Turn y Past and Present (p.17). En general los autores coinciden con Chartier en la importancia de la lengua dado que la cantidad de obras citadas en los diferentes capítulos dan cuenta de que "la historia, sea o no cultural, afortunadamente se escribe todavía en lenguas tan numerosas que ningún lector puede saberlas todas" (p. 245). Hay una enorme variedad de libros de la materia a los que los especialistas no pueden acceder por no conocer las lenguas en las que fueron escritos. ${ }^{14}$ Como alternativa a esta solución, el inspirador de la obra propone la lengua inglesa como vector del modelo anglosajón en pos de la desnacionalización de la disciplina histórica (p. 18). Más allá de la lengua, existen intercambios personales entre historiadores de distintos países que facilitan las transferencias culturales. Por último, Poirrier declara que la aparición de este volumen es poseedora de una intencionalidad concreta: permitir un mejor conocimiento de las distintas formas de la historia cultural a fin de que el método comparativo motive el dialogo y se produzca la definitiva superación de los provincialismos y etnocentrismos historiográficos (p.20).

Ezequiel Borgognoni

Universidad de Buenos Aires. CONICET. eborgognoni@filo.uba.ar

Fecha de recepción: 17 de julio de 2015.

Fecha de aceptación: 30 de julio de 2015.

Publicación: 31 de diciembre de 2015.

Para citar este artículo: Ezequiel Borgognoni, "Philippe Poirrier (ed.), La Historia Cultural ¿un giro historiográfico mundial? Valencia: Universidad de Valencia, 2012, 252 págs.”, Historiografías, 10 (julio-diciembre, 2015): pp. 144-149.

http://www.unizar.es/historiografias/historiografias/numeros/10/borgognoni.pdf

14 A modo de ejemplo, véanse los capítulos de Palle Ove Christianses y Ecaterina Lung referidos a Escandinavia y Rumania, respectivamente.

ISSN 2174-4289 